\title{
Programa de ejercicio físico para mejorar fuerza muscular en personas sedentarias*
}

\author{
Laura Elizabeth Castro* \\ Laura Teresa Salazar Solis ${ }^{* * *}$ \\ Karen Girleida Perea Valderrama***
}

Recibido: febrero 14 de 2013 • Evaluado: marzo 22 de 2013

Aceptado: mayo 28 de 2013

\section{Resumen}

El sedentarismo es una realidad social dada por el ritmo de vida actual, un problema que genera la aparición de riesgos cardiovasculares y la posibilidad de sufrir una enfermedad grave. El objetivo de este estudio fue conocer la efectividad de un programa de ejercicio físico para mejorar la fuerza muscular en personas sedentarias. Se realizó un estudio cuasiexperimental, en el cual se aplicó un programa de ejercicios 5 días a la semana por 4 semanas a un grupo de 60 individuos sedentarios. Se hizó pretest y postest de fuerza muscular, flexibilidad y composición corporal. Al finalizar el estudio se encontró mejoría en los parámetros evaluados.

Palabras clave: ejercicio físico, factor de riesgo cardiovascular, fuerza muscular, sedentarismo.

" Resultado de investigación. Estudio realizado en la Universidad Manuela Beltrán, Sede Bogotá. Tiempo de realización: año 2011.

** Magister en Salud Pública de la Universidad Nacional de Colombia. Grupo de investigación Ejercicio físico y deporte, Universidad Manuela Beltrán, (Colombia). Autor. Correo: lauraunica12@gmail.com

*** Fisioterapeuta, Universidad Manuela Beltrán, (Colombia). Co-autor. Correo: laura. s.s89@hotmail.es

*** Fisioterapeuta, Universidad Manuela Beltrán, (Colombia). Co-autor. Correo: kagipe 20@hotmail.com 


\title{
Physical exercise program to improve muscle strength in sedentary people
}

\begin{abstract}
Physical inactivity is a social reality given by the pace of life that takes today, still a problem generating the appearance of cardiovascular risk and therefore the chance of getting a disease so detrimental to the quality of life of people. The aim of this study was to determine the effectiveness of an exercise program to improve muscle strength in sedentary people. We conducted a quasi-experimental study in which we applied an exercise program five days a week for 4 weeks even group of 60 sedentary individuals. Pretest and posttest of muscle strength, flexibility and body composition. At the end of the study found improvement in parameters evaluated.
\end{abstract}

Keywords: Exercise, cardiovascular risk factor, muscle strength, physical inactivity. 


\section{Introducción}

Existe un aumento en la presencia de factores de riesgo para la salud, derivados del cambio de estilo de vida generado por el desarrollo económico, político y social de las poblaciones; dentro de estos se encuentran la obesidad, el consumo de sustancias psicoactivas y alcohólicas, y el sedentarismo, relacionado con las causas de patologías como hipertensión, diabetes e infarto agudo de miocardio (Varo, 2003; Tribess, 2009).

El sedentarismo es una realidad social ocasionada por el ritmo de vida que se lleva en la actualidad debido a la evolución en los sistemas de transporte, los avances tecnológicos en cuanto a las máquinas de utilización laboral, los videojuegos, el internet, las computadoras y los controles remotos. El sedentarismo genera la aparición de riesgos cardiovasculares, aumentando la posibilidad de sufrir una enfermedad cardiovascular produciendo disminución de la calidad de vida y un aumento en la tasa de mortalidad (Sánchez, Moreno, Marín, y García, 2009).

La Organización Mundial de la Salud (OMS) reportó que en 2008 murieron por enfermedad cardiovascular 17,3 millones de personas, lo cual representa un $30 \%$ de todas las muertes registradas en el mundo; 7,3 millones se debieron a la cardiopatía coronaria, y 6,2 millones a los accidentes cerebro-vasculares (AVC). Las muertes por enfermedad cerebro-vascular (ECV) afectan por igual a ambos sexos, y más del $80 \%$ se producen en países de ingresos bajos y medios. Se calcula que en 2030 morirán cerca de 25 millones de personas por ECV, sobre todo por cardiopatías y accidentes cerebro-vasculares, y se prevé que sigan siendo la principal causa de muerte (OMS, 2012).

Moya-Sifontes et al. (2006) estudiaron a 314 estudiantes universitarios venezolanos entre 17 y 29 años de edad, y reportaron que un 31,5\% eran sedentarios, $47,5 \%$ eran suficientemente activos y tan solo $21,02 \%$ tenían un nivel de actividad física elevado. Los resultados del estudio fueron similares a los encontrados en Chile y en la población colombiana (Romo y Cerda, 2002). Chacón, González, Velásquez y Segura (2008) mencionan que el estilo de vida caracterizado por hábitos sedentarios provoca un cuerpo débil, por lo que la acción del ejercicio sobre el sistema cardiovascular es doble: actúa disminuyendo ciertos factores de riesgos, y sobre el músculo cardíaco y las arterias coronarias. Promueve el desarrollo de la circulación 
colateral, eleva niveles de colesterol HDL y disminuye el colesterol total y los triglicéridos.

El sedentarismo se ha constituido en un riesgo cardiovascular que va en aumento en Colombia y en el mundo, según la Organización Panamericana de la Salud (OPS, 2010), tres cuartos de la población tienen un estilo de vida sedentario. La falta de actividad está relacionada de forma directa con enfermedades cardiovasculares, la obesidad y la diabetes, tres de los principales problemas de salud del mundo de hoy (Universidad del Rosario, 2010).

El sedentarismo es uno de los causantes de que las personas reduzcan progresivamente su fuerza muscular a razón del no acondicionamiento físico permanente que se presenta por la ausencia de la práctica de actividad física, teniendo en cuenta que las personas que se mantienen en constante reposo presentan un gasto energético bajo, lo que origina una acumulación excesiva de calorías en el cuerpo por el poco consumo energético, y porque la inactividad no va acompañada de una disminución en la ingesta calórica, se convierte en grandes depósitos de grasas y provoca una mayor demanda en el funcionamiento del organismo principalmente en el sistema cardiovascular donde se tiene bajo gasto cardíaco, aumento en la tensión arterial y la frecuencia cardíaca. Para un equilibrio sistémico y metabólico debe existir una proporción adecuada entre el consumo de nutrientes ricos en calorías y gastos calóricos, que se obtiene mediante la práctica habitual de ejercicio físico (García, García Pérez y Bonet, 2007).

El sedentarismo es un factor de riesgo exógeno cambiable que se puede mejorar mediante un programa de ejercicio físico, el cual sirve de estímulo para las personas que quieren transformar su estilo de vida y de esa manera tener un buen estado de salud, teniendo en cuenta que estos estilos de vida están influenciados por factores socio demográficos como el sexo, edad, estudios, profesión e incluso estado civil (Cuevas, 2003).

El sedentarismo se ha convertido en un gran problema debido a que los avances tecnológicos limitan la movilidad de las personas, con más facilidad se pueden realizar transacciones o pagos de recibos desde un asiento mediante la internet, además, la rutina de transporte privado disminuye el uso de los grandes grupos musculares y por consiguiente un mínimo desgaste energético de los mismos (Cabrera, 2007). En este mismo sentido Blasco, citado por López-Miñarro (2009: 4), expresa: 
A medida que la tecnología ha permitido liberar al hombre de las tareas pesadas que requerían grandes dosis de esfuerzo físico, así como facilitarle los desplazamientos sin tener que caminar, la sociedad occidental ha modelado unos quehaceres cotidianos que no requieren, en la mayor parte de los casos, prácticamente de ningún esfuerzo físico, ni tampoco de desplazamientos que deban hacerse andando.

Casimiro (1999) indica que los importantes cambios producidos en los últimos años en los ámbitos socioeconómico y cultural, y los numerosos avances tecnológicos, han permitido a las sociedades desarrolladas cambiar sus empleos, abandonándose gran parte de los trabajos en los que había que emplear la fuerza física. Además de dicha mecanización del trabajo, se han automatizado las tareas domésticas, se han reducido las dimensiones de la vivienda, han disminuido los juegos populares y tradicionales de las calles, sustituyéndose por videoconsolas y consumo de televisión; por último, el fenómeno de la informática ha favorecido un aumento del sedentarismo de la población, tanto en la jornada laboral como en sus desplazamientos y en el tiempo libre.

Cuando hay inactividad física la fuerza muscular se va perdiendo lentamente, lo que a largo plazo le impide al individuo ejecutar acciones donde sea necesaria la utilización de esta, con consecuencias en el organismo como: debilidad en los tejidos conectivos, bajo funcionamiento del músculo cardíaco, lo que implica una mayor resistencia de la sangre en las arterias originando hipertensión arterial, los huesos no tienen una adecuada absorción de nutrientes predisponiendo al individuo a sufrir de osteoporosis, alteraciones en el equilibrio y la coordinación (Barrera, 2000).

Es por eso que se evidenció la necesidad de generar un programa de ejercicio físico para personas sedentarias, en el cual se haga énfasis en la fuerza muscular; planteándose como objetivo de investigación conocer la efectividad de un programa de ejercicio físico para el mejoramiento de la fuerza muscular en pacientes sedentarios.

\section{Metodología}

El presente estudio responde a un diseño cuantitativo de tipo cuasiexperimental. En cuanto a la población los criterios de inclusión fueron: personas sedentarias, hombres y mujeres entre 16 y 59 años, y los criterios de 
exclusión: personas que tuvieran diabetes, hipertensión, dislipidemia, fumadores, mujeres embarazadas o con alguna patología concomitante, que presentaran alguna discapacidad física o mental o que no firmarán el consentimiento informado. El tamaño de muestra fue de 60 personas. El programa de ejercicio se construyó en un estudio previo de revisión sistemática y se aplicó por un mes teniendo en cuenta una evaluación fisioterapéutica inicial y otra final para identificar los cambios musculo-esqueléticos que se evidenciaron después de la ejecución del programa que tuvo énfasis en la fuerza muscular. Las evaluaciones realizadas fueron de fuerza muscular a través de dinamometría de miembros superiores e inferiores, flexibilidad con Sit and Reach y composición corporal.

La información de la evaluación pre y pos se almacenó en una matriz, para un análisis que se hizo por medio de estadística descriptiva a través de varianza, covarianzas, moda, medias y comprobación de hipótesis a través de la prueba t.

El programa fue realizado por Barrera y Rodríguez (2010), y tiene como fin mejorar la fuerza muscular en pacientes sedentarios. Este programa se realizó durante un mes con una frecuencia de cinco veces por semana, duración de 40 minutos, e intensidad progresiva de 35 a $80 \%$ de una $1 \mathrm{RM}$. Consta de varios componentes entre ellos un calentamiento que incluye cinesiterapia activa para mejorar la movilidad articular en cuello, hombros, muñecas, caderas, rodillas y cuello de pie, seguido por aeróbicos para el control de la respiración y posteriormente estiramientos activos para evitar las retracciones y lesiones durante el entrenamiento y por último, lo primordial: el fortalecimiento muscular.

\section{Resultados}

Se realizó la aplicación del programa de entrenamiento físico con el fin de mejorar la fuerza muscular bajo los siguientes parámetros; duración de un mes, con frecuencia de cinco días a la semana y durante 40 minutos la sesión. El tamaño de muestra fue de 60 personas sedentarias a las cuales se les aplicó una evaluación fisioterapéutica inicial y final. En el transcurso de la ejecución del programa de entrenamiento desertaron cinco individuos, mientras 
que seis no asistieron a la evaluación final; por esta razón, se analizaron los datos de 49 individuos que finalizaron satisfactoriamente el programa, donde el 55,1 \% son hombres y el 44,9\% mujeres, con una edad promedio de 17 años, con rango de edad de (16-22 años), aunque en los criterios de inclusión se puso una edad límite de 59 años, las personas que cumplieron con todos los criterios de inclusión estaban en este rango de edad, el resto fue excluido por tener alguna enfermedad concomitante, no firmar el consentimiento informado o tener el tiempo para participar en el estudio.

Las evaluaciones constaban de historia clínica, evaluación de fuerza muscular a través de dinamometría de miembros superiores e inferiores, flexibilidad con Sit and Reach y composición corporal. Los datos obtenidos a partir de la realización de las evaluaciones fisioterapéuticas se almacenaron en una base de datos en Excel; para analizar y conocer los resultados, se estudiaron la media, mediana y moda de cada dato.

Se encontró una media inicial para dinamometría de miembros superiores en $71.836 \mathrm{~kg} / \mathrm{f}$, miembros inferiores $164.938 \mathrm{~kg} / \mathrm{f}$, índice de masa corporal 21.660 y en el test de Sit and Reach $17.551 \mathrm{~cm}$, como se muestra en la tabla 1.

Tabla 1. Datos iniciales

\begin{tabular}{lcccc}
\hline & $\begin{array}{c}\text { Índice } \\
\text { de masa } \\
\text { corporal }\end{array}$ & $\begin{array}{c}\text { Dinamometría } \\
\text { de MMSS }\end{array}$ & $\begin{array}{c}\text { Dinamometría } \\
\text { de MMII }\end{array}$ & $\begin{array}{c}\text { Test de Sit And } \\
\text { Reach }\end{array}$ \\
\hline Sumatoria & 1.061 .328 & $3520 \mathrm{~kg} / \mathrm{f}$ & $8082 \mathrm{~kg} / \mathrm{f}$ & $860 \mathrm{~cm}$ \\
Media & 21.660 & $71,8367347 \mathrm{~kg} / \mathrm{f}$ & $164,938776 \mathrm{~kg} / \mathrm{f}$ & $17,5510204 \mathrm{~cm}$ \\
Mediana & 20,699 & $65 \mathrm{~kg} / \mathrm{f}$ & $140 \mathrm{~kg} / \mathrm{f}$ & $18 \mathrm{~cm}$ \\
Moda & 21,093 & $60,65 \mathrm{~kg} / \mathrm{f}$ & $100 \mathrm{~kg} / \mathrm{f}$ & $23,25 \mathrm{~cm}$ \\
\hline
\end{tabular}

Fuente: elaboración propia (2011).

Dinamometría de miembro superior. Se obtuvo una ganancia de 625 $\mathrm{kg} / \mathrm{f}$ al final de la aplicación del programa en los pacientes, con una evaluación inicial de $3520 \mathrm{~kg} / \mathrm{y}$ una final de $4145 \mathrm{~kg} / \mathrm{f}$ y un logro en la media de $12,755102 \mathrm{~kg} / \mathrm{f}$ (ver figura 1). 
Figura1. Dinamometría de miembro superior

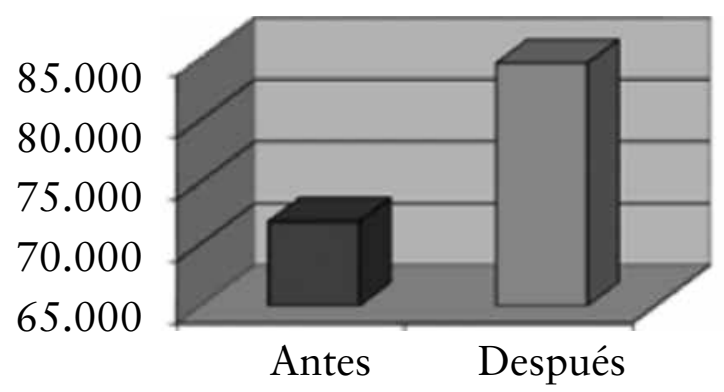

Fuente: elaboración propia (2011).

Dinamometría de miembro inferior. Se obtuvo una ganancia de 1668 $\mathrm{kg} / \mathrm{f}$ al final de la aplicación del programa en los pacientes, con una evaluación inicial de $8082 \mathrm{~kg} / \mathrm{f}$ y una final de $9750 \mathrm{~kg} /$ fuerza y un logro en la media de $34,0408163 \mathrm{~kg} / \mathrm{f}$ (ver figura 2).

Figura 2. Dinamometría de miembro inferior

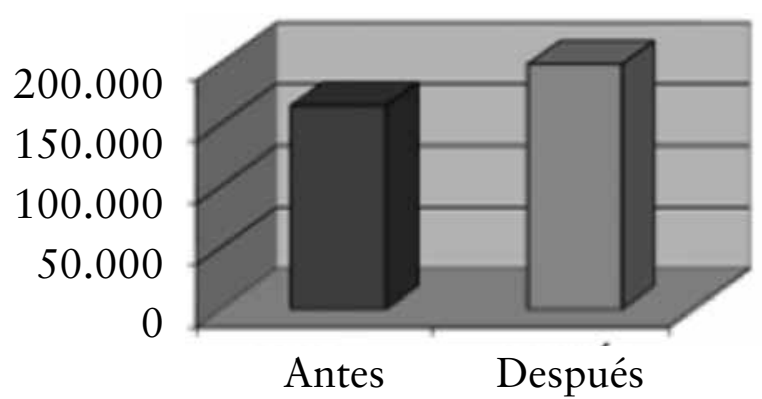

Fuente: elaboración propia (2011). 
Test de Sit and Reach. Se obtuvo una ganancia de $14,1 \mathrm{~cm}$ al final de la aplicación del programa en los pacientes, con una evaluación inicial de $8,60 \mathrm{~cm}$ y una final de $10,01 \mathrm{~cm}$ y un logro en la media de 2,87755102 (ver figura 3). Aunque el programa tenía énfasis en fuerza muscular, dentro de las sesiones una parte del entrenamiento estuvo destinado a mejorar la flexibilidad.

Figura 3. Test de Sit and Reach.

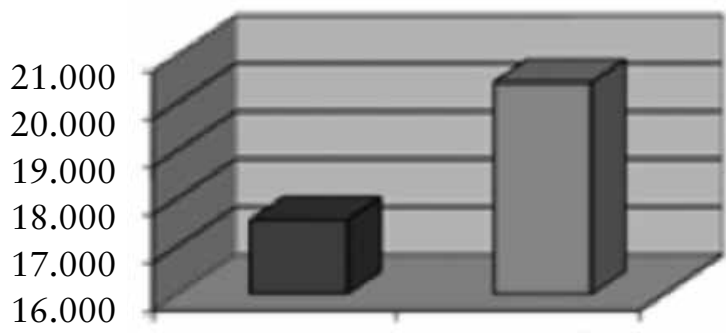

Fuente: elaboración propia (2011).

Índice de masa corporal. Dentro de los ítems de evaluación se contempló la composición corporal a través del índice de masa corporal, donde se encontró una diferencia mínima entre la evaluación inicial y la final, pero no podemos inferir que este cambio sea dado por el programa de entrenamiento, ya que no se controlaron otras variables que pudieron inferir para este cambio. En la evaluación inicial los valores de la media fueron de 21,66 $\mathrm{kg} / \mathrm{mts}^{2}$, y en la final fue de $21,7 \mathrm{~kg} / \mathrm{mts}^{2}$.

En la tabla 2 se describen los resultados finales de dinamometría de miembros superiores e inferiores, test de Sit and Reach e índice de masa corporal, donde se encontraron cambios favorables en cada una de las variables. 
Tabla 2. Datos finales

\begin{tabular}{lcccc}
\hline & $\begin{array}{c}\text { Índice de } \\
\text { masa corporal }\end{array}$ & \multicolumn{2}{c}{ Dinamometría } & Dinamometría de \\
de MMSS & $\begin{array}{c}\text { Test de Sit } \\
\text { And Reach }\end{array}$ \\
\hline Sumatoria & 1.063 .553 & 4145 & 9750 & 1001 \\
Media & 21.705 & 84,5918367 & 198,9795918 & 20,4285714 \\
Mediana & 20.957 & 75 & 180 & 21 \\
Moda & $20957,21093,23191$ & 60 & $110,120,160,220$ & 28 \\
\hline
\end{tabular}

Fuente: elaboración propia (2011).

Para responder a la pregunta de investigación se planteó realizar una prueba $t$, la cual mostró que se rechazó la hipótesis nula, mostrando la eficacia del programa de ejercicio. Ver tabla 3.

Tabla 3. Dinamometría de miembro inferior

\section{Prueba t para dinamometría de miembro inferior}

Suma de las puntuaciones pretratamiento:

8082

Suma de las puntuaciones postratamiento:

9750

Resultado (diferencia):

1668

Resultado ${ }^{2}$ :

125732

Número de individuos:

Media postratamiento: 198,9795918

Media pretratamiento: 164,938776

PRUEBA t: 7.03

Fuente: Elaboración propia (2011).

\section{Discusión}

El programa de ejercicio físico que se realizó durante la investigación tenía el propósito de aumentar la fuerza muscular en pacientes sedentarios, el diseño se apoyó en una investigación previa de los principios de la sobrecarga 
progresiva, en donde se aumentó a los pacientes, progresivamente, la carga de trabajo, para que existiera un aumento de la fuerza muscular. Por esto los músculos de los miembros superiores e inferiores fueron sometidos a cargas mayores de las acostumbradas a realizar cotidianamente, de esta forma habría un mayor reclutamiento de fibras musculares tras la resistencia. Inicialmente, se realizó una evaluación en donde se tuvieron en cuenta los siguientes ítems: fuerza muscular en miembros superiores e inferiores, flexibilidad, e índice de masa corporal, posteriormente aplicado el programa, se hizo la evaluación final.

La investigación encontró que el programa de ejercicio físico mejoró la fuerza muscular en pacientes sedentarios, en miembros superiores e inferiores; lo que demuestra que las personas sedentarias pueden mejorar sus cualidades físicas al practicar una actividad física (López-Miñarro, 2009). Como lo mencionan González y Vaquero (2000), estamos acostumbrados a asociar el ejercicio físico con actividades lúdicas, de baja o nula intensidad en las que el carácter lúdico es el principal protagonista de la sesión dejando atrás el rendimiento físico. Un correcto trabajo de fuerza bien realizado, a unas cargas de entrenamiento considerables, produce una mejora fisiológica que aumenta su capacidad funcional, y que mejora sus condiciones de vida y disminuye factores de riesgo.

Araya et al. (2012) describe resultados similares en un grupo de mujeres mayores de 60 años, con un programa de ejercicio basado en fuerza muscular; los resultados obtenidos muestran un efecto positivo sobre el rendimiento de todos los test físicos $(\mathrm{p}<0,05)$. Los parámetros antropométricos mostraron una reducción en el índice cintura-cadera $(\mathrm{p}=0,029)$ y del perímetro de cintura $(\mathrm{p}=0,014)$, sin que el resto de las variables analizadas mostraran cambios de interés. Entre las asociaciones observadas, el test de salto vertical mostró una correlación negativa con el test de equilibrio y con el test de sentarse y levantarse, antes y después del tratamiento $(R=-0,495$; $R=-0,699$ en el PREtest y $R=-0,373 ; R=-0,463$ en el POST para la relación CMJ y Equilibrio y CMJ y Ln S-L test respectivamente, $\mathrm{P}<0,05)$. Concluyendo que el programa mejora la capacidad física (equilibrio, fuerza de prensión manual, fuerza de piernas y flexibilidad anterior de tronco) y los parámetros antropométricos (índice cintura-cadera y perímetro de cintura).

Coto (2006) señala que con un programa de ejercicio con énfasis en fuerza muscular, los resultados indican que en la variable memoria auditiva 
y discriminación perceptual se presentaron mejores resultados en las condiciones del $30 \%$ y $70 \%$ de 5 Repeticiones Máximas (5RM) al compararlas con la condición control. Con lo que respecta a las mediciones se presentó una mejoría en las condiciones del $30 \%$ y $70 \%$, inmediatamente después de terminado el ejercicio, al compararlo con el pretest. El efecto agudo se mantuvo a los $30 \mathrm{~min}$ y a los $60 \mathrm{~min}$, de finalizado.

Para Calahorro, Torres, Lara y Zagalaz (2011) el trabajo de flexibilidad previene lesiones y mejora el rendimiento, con el fin de evitar sobrecargas y acortamientos musculares en la práctica física, al aumentar la flexibilidad mejora la fuerza muscular. Según Morehouse y Miller, citados por Hoeger (2005), existe una relación directa entre el consumo de oxígeno como resultado de la actividad metabólica y la cantidad de peso corporal magro, a medida que aumenta el tamaño muscular, así lo hace el metabolismo en reposo; o la cantidad de energía (expresada en calorías), requerida por un individuo durante condiciones de reposo para sostener adecuadamente las funciones celulares.

El entrenamiento de la fuerza muscular activa los vasos sanguíneos, permitiendo que los nutrientes lleguen hasta el último rincón de la fibra muscular y una eliminación adecuada de las sustancias de desechos, todo gracias al trabajo muscular. El músculo tendrá una mayor conducción nerviosa y una mayor velocidad de contracción, lo que indica un mayor reclutamiento de las fibras, alcanzando una adecuada biomecánica entre los músculos agonistas y antagonistas.

El fortalecimiento muscular también aumenta los niveles de VO2 máximo, como lo hacen los ejercicios aeróbicos, claro que en menor medida, lo que significa que también pueden beneficiar al funcionamiento cardiopulmonar, evitando la concentración de tejido adiposo corporal, disminuyendo el riesgo de que un ateroma o embolo viaje al torrente sanguíneo, impidiendo un posible accidente cerebrovascular, siendo esto lo que se quiere frenar, para que hayan menos enfermedades, discapacidades y muertes por esta causa. Lo que se desea es mejorar la calidad de vida de los pacientes, en donde gocen de buena salud (García et al., 2010; Águila, Vicente, Llaguno, Sánchez y Costa, 2012; Estévez, Tercedor y Delgado, 2012).

Los ejercicios encaminados al fortalecimiento muscular, utilizados para el acondicionamiento físico y que tiene la finalidad de aumentar la fuerza, también pueden ser empleados con otros fines. En la medida en que 
cumplen su objetivo principal, favorecen la ejecución adecuada de otros procesos corporales, que benefician la salud del ser humano, previniendo las enfermedades más frecuentes de los últimos tiempos.

El programa de ejercicio físico fue efectivo porque mejoró la fuerza muscular en personas sedentarias siendo este el fin principal de investigación. Como segundo factor, al aumentar la fuerza muscular en estos individuos se disminuye el factor de riesgo cardiovascular.

\section{Conclusiones}

El programa de ejercicio físico para mejorar la fuerza muscular en personas sedentarias, arrojó resultados positivos puesto que los individuos que participaron en el programa tuvieron un incremento de la fuerza muscular como lo indica la media pretratamiento de dinamometría para miembro superior equivalente a $71.836 \mathrm{~kg} /$ peso y el resultado postratamiento fue de 84.591 $\mathrm{kg} /$ peso. En miembros inferiores la media pretratamiento fue de $164.938 \mathrm{~kg} /$ peso y postratamiento $198.97 \mathrm{~kg} /$ peso, además de eso se obtuvieron otros resultados como el aumento de la flexibilidad, y un cambio pequeño en el índice de masa corporal, esta último no se puede asegurar que sea producto del programa de ejercicio ya que no se controlaron todas las variables que influyen para este cambio. Se recomienda implementar el programa de ejercicio físico en otras localidades de Bogotá como medio de prevención a la salud en personas sedentarias puesto que al aumentar la fuerza muscular también disminuye, de alguna manera, el factor de riesgo cardiovascular.

\section{Referencias}

Águila, Y., Vicente, B.V., Llaguno, G.A., Sánchez, J.F. y Costa, M. (2012). Effect of physical exercise on metabolic control and risk factors in patients with type 2 diabetes mellitus: a quasi-experimental study. Medwave, 12 (10). Recuperado de http://www.medwave.cl/link.cgi/Medwave/Estudios/Investigacion/5547

Araya, S., Padial, P., Feriche, B., Gálvez, A., Pereira, J. y Mariscal-Arcase, M. (2012). Incidencia de un programa de actividad física sobre los parámetros antropométricos y la condición física en mujeres mayores de 60 años. Nutrición

Vol. 3, N. ${ }^{\circ}$ 6, julio-diciembre de 2013, pp. 13-27 
Hospitalaria, 27 (5), 1472-1479. Recuperado de: http://www.nutricionhospitalaria.com/pdf/5899.pdf

Barrera, S.G. y Rodríguez, A. L. Características de la fuerza muscular en pacientes con sedentarismo como factor de riesgo cardiovascular. Bogotá: Universidad Manuela Beltrán, p.1-97.

Barrera, E. (2000). Conocimientos y factores de riesgo cardiovascular y su relación con la presencia de la hipertensión. Colombia Medica, 31 (1).

Cabrera, L. (2007). Sedentarismo: tiempo de ocio activo frente al porcentaje de gasto energético. Revista Española de Cardiología, 60 (3), 244-250.

Calahorro, F., Torres, G., Lara, A., y Zagalaz, M. L. (2011). Parameters related to the competition's physical training. Journal of Sport and Health Research. 3 (2), 113-128.

Casimiro, A.J. (1999). Comparación, evolución y relación de hábitos saludables y nivel de condición física-salud en escolares, al finalizar los estudios de educación primaria (12 años) y de educación secundaria obligatoria (16 años). Tesis Doctoral. Universidad de Granada.

Chacón, D., González, R. M., Velásquez, G. A. y Segura, O. (2008). Pesquisa de factores de riesgos asociados a la hipertensión arterial. Risk Factors Analysis Associated to Hypertension. Recuperado de http://www.cocmed.sld.cu/no121/ n121ori8.htm

Coto, E. y Rivera, C. (2006). Efecto agudo en los procesos cognitivos en adultos mayores al realizar ejercicio de fuerza a diferentes intensidades. Revista InterSedes, (8), 1-12. Recuperado de http://www.intersedes.ucr.ac.cr/ojs/index.php/intersedes/article/viewFile/89/88

Cuevas, F.J. (2003). Percepción de la calidad de vida de los pacientes hipertensos: factores influyentes. Curso 2003/04, Ciencias y Tecnologías/6. Servicios y publicaciones, Universidad de La Laguna, España. Recuperado de http://dialnet. unirioja.es/servlet/tesis?codigo $=955$

Estévez-López, F., Tercedor, P. y Delgado-Fernández, M. (2012). Recomendaciones de actividad física para adultos sanos. Journal of Sport and Health Research. 4 (3), 233-244. Recuperado de http://www.journalshr.com/papers/Vol\%20 4_N\%203/V04_3_3.pdf

García, R.M.; García Pérez y Bonet, M. (2007). Sedentarismo y su relación en la calidad de vida relativa a la salud. Revista Cubana de Higiene y Epidemiología, 45(1).

García-Ortiz, L., Grandes, G., Sánchez-Pérez, A., Montoya, I., Iglesias-Valiente, J., Recio-Rodríguez, J., Castaño-Sánchez, Y. y Gómez-Marcos, M. (2010). Efecto 
en el riesgo cardiovascular de una intervención para la promoción del ejercicio físico en sujetos sedentarios por el médico de familia. Revista Española de Cardiología, 63 (11). Recuperado de http://www.revespcardiol.org/es/ efecto-el-riesgo-cardiovascular-una/articulo/13183605/

González, J. M. y Vaquero, M. (2000). Indicaciones y sugerencias sobre el entrenamiento de fuerza y resistencia en ancianos. Revista Internacional de Medicina y Ciencias de la Actividad Física y el Deporte, 1 (1), 10-26. Recuperado de: http://cdeporte.rediris.es/revista/revista1/ancianos.htm

Hoeger, B. (2005). Educación física de base. Universidad de los Andes. MéridaVenezuela, 61-63.

López-Miñarro, P.A. (2009). La salud y la actividad física en el marco de la sociedad moderna. Facultad de Educación. Universidad de Murcia.

Moya-Sifontes, M., García, P., Lucena, N., Casañas, R., Brito, P., Rodríguez, A., Flórez, Z. y Cordero, R. (2006). Hipocinetismo: ¿un problema de salud entre jóvenes ucevistas? Revista de la Facultad de Medicina, 29 (1), 74-79.

Organización Mundial de la Salud (2012). Enfermedades cardiovasculares. Recuperado de http://www.who.int/mediacentre/factsheets/fs317/es/index. html

Organización Panamericana de la Salud. (2010). La inactividad física: Un factor principal de riesgo para la salud en las Américas. Recuperado de http://www1. paho.org/Spanish/HPP/HPN/whd2002-factsheet3.pdf

Romo, R. y Cerda, M., (2002). Estilo de vida en los estudiantes universitarios de la Facultad de Medicina de la Universidad de Chile. Revista de Salud Pública, 7 (11), 14-25.

Sánchez, M., Moreno, G., Marín, M. y García, L. H. (2009). Factores de riesgo cardiovascular en poblaciones Jóvenes. Revista de Salud Pública, 11 (1), 110-122.

Tribess, S. (2009). Factores asociados con la inactividad física en mujeres ancianas en las comunidades de bajos ingresos. Revista de salud pública, 11 (1).

Universidad del Rosario (2010). Muévase contra el sedentarismo, fascículo 7.

Varo, J. (2003). Beneficios de la actividad física y riesgos del sedentarismo. Barcelona-España, Med clin 121 (17), 665-672. 